\title{
Recombinant cold shock domain containing protein is a potential antigen to detect specific antibody during early and late infections of Haemonchus contortus in goat
}

Muhammad Ali-ul-Husnain Naqvi, Kalibixiati Aimulajiang, Muhammad Ali Memon, Muhammad Waqqas Hasan, Sana Zahra Naqvi, Shakeel Ahmed Lakho, Wen Chu, Lixin Xu, Xiaokai Song, Xiangrui Li and Ruofeng Yan * (D)

\begin{abstract}
Background: Haemonchus contortus (H. contortus) is one of the most important parasites that cause huge economic losses to small ruminant industry worldwide. Effective prognosis and treatment depend upon the early diagnosis of $H$. contortus infection. To date, no widely-approved methods for the identification of prepatent $H$. contortus infection are available to identify prepatent $\mathrm{H}$. contortus infection properly. The aim of this study was to evaluate the diagnostic potential of recombinant cold shock $\mathrm{H}$. contortus protein (rHc-CS) during early and late infections of $\mathrm{H}$. contortus in goat.

Results: Purified rHc-CS exhibited a clear band, with a molecular weight about $38 \mathrm{kDa}$. H. contortus eggs were not detected by fecal egg count technique from feces collected at 0 to 14 days post infection (D.P.I). However, eggs were detected at 21, 28 and 35 D.P.I. Hence, results of immunoblotting assay showed specific anti rHc-CS antibody detection in all goat sera collected at early stage (14 D.P.I) and late stage (21-103 D.P.I) of H. contortus infection. Furthermore, no cross reactivity was observed against Trichinella spiralis, Fasciola hepatica and Toxoplasma gondii or uninfected goats. Among several evaluated $\mathrm{rHc}$-CS indirect-ELISA format variables, favorable antigen coating concentration was found $0.28 \mathrm{\mu g} /$ well at $37^{\circ} \mathrm{C} 1 \mathrm{~h}$ and overnight at $4{ }^{\circ} \mathrm{C}$. Moreover, optimum dilution ratio of serum and rabbit anti-goat lgG was recorded as 1:100 and 1:4000, respectively. The best blocking buffer was 5\% Bovine Serum Albumin (BSA) while the best time for blocking, serum incubation and TMB reaction were recorded as 60, 120 and $10 \mathrm{~min}$, respectively. The cut-off value for positive and negative interpretation was determined as 0.352 $\left(\mathrm{OD}_{450}\right)$. The diagnostic specificity and sensitivity of the $\mathrm{rHc}-\mathrm{CS}$, both were recorded as $100 \%$.
\end{abstract}

Conclusion: These results validated that $\mathrm{rHc}-\mathrm{CS}$ is a potential immunodiagnostic antigen to detect the specific antibodies during early and late $H$. contortus infections in goat.

Keywords: Haemonchus contortus, Early diagnosis, Cold shock domain containing protein, Immunoblotting, Indirect-ELISA

\footnotetext{
* Correspondence: yanruofeng@njau.edu.cn

MOE Joint International Research Laboratory of Animal Health and Food

Safety, College of Veterinary Medicine, Nanjing Agricultural University, No.1,

Weigang, Nanjing, Jiangsu Province, People's Republic of China 210095
}

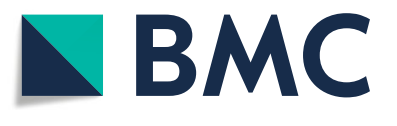

(c) The Author(s). 2020 Open Access This article is distributed under the terms of the Creative Commons Attribution 4.0 International License (http://creativecommons.org/licenses/by/4.0/), which permits unrestricted use, distribution, and reproduction in any medium, provided you give appropriate credit to the original author(s) and the source, provide a link to the Creative Commons license, and indicate if changes were made. The Creative Commons Public Domain Dedication waiver (http://creativecommons.org/publicdomain/zero/1.0/) applies to the data made available in this article, unless otherwise stated. 


\section{Background}

Haemonchus contortus ( $H$. contortus) is an important haematophagous gastrointestinal parasite of small ruminants. It can suck about $0.05 \mathrm{~mL}$ blood per day $[1,2]$ and may cause acute anemia, edema, diarrhea, severe frailty weight loss, and ultimately death [3, 4]. Due to high mortality and morbidity rate, $H$. contortus infection causes significant economic losses to small ruminants particularly in humid, tropical and subtropical regions $[5,6]$. China mainly contributes $17.3 \%$ of world's total goat population [7] in which different prevalence rate of $H$. contortus infection has been reported in several provinces [8]. The control of this parasite mainly relies on accurate and early diagnosis. Conventional fecal egg counts technique is main method to diagnose this infection clinically but it is difficult to detect $H$. contortus eggs in feces before 21-25 days of infection [4]. Last larval stages of this parasite feed on blood [9] and may suck up to $1 / 5$ th of total circulating erythrocyte volume in young animal [10]. H. contortus blood feeding starts at 11th day of infection [11] but clinical signs usually become apparent when infection becomes severe [12]. Another way for the diagnosis of this infection depends on the degree of anemia using FAMACHA system in which an ocular conjunctiva color chart is used for assessment of anemia to decide which animal requires treatment for $H$. contortus infection [13]. However, these methods are often nonspecific, insensitive, laborious, time consuming [14] and most importantly lacking the ability to detect the infection at early stage. Hence, early detection of $H$. contortus is crucial and necessary to control infection effectively [15].

During early infection, parasites produce and release Excretory and Secretory Products (ESPs) that play an important immunological role [16]. ESPs have been widely used as diagnostic antigen because these products have good specificity and sensitivity [17]. ESPs contain numerous proteins which depress the immunity of host at prepatent stages of infection by modulating immune system [18]. Recently, immunoblotting and ELISA based on different types of antigens (somatic and crude) have been reported for the detection of $H$. contortus specific antibodies [4, 15, 19, 20]. However, shared antigenic composition is major disadvantage of these antigens that leads to cross-reactivity in the diagnosis of $H$. contortus infection [21]. Currently, there is a lack of potential immunogenic antigen which can accurately detect the particular infectious stage of this helminth in goat. To overcome these challenges and to improve control strategies, a potential antigen based immunodiagnostic assay is needed [15].

Cold shock domain is present in every cellular compartment and it is a constituent part of nearly all prokaryotes and eukaryotes. In animals, cold shock proteins exhibit broad functions that relate to the growth and development of a cell. These proteins have special ability to bind with nucleic acid to regulate not only their own expression but also involve in the regulation of virulent genes [22]. In our previous proteomic study, interaction of $H$. contortus (Hc)ESPs with host peripheral blood cells at different developmental stages was reported. The Cold Shock domain containing protein (CS) is one of these HcESPs, that binds to goat PBMCs at $\mathrm{L}_{4}$ and $\mathrm{L}_{5} H$. contortus development stages [23]. Hence the presence of CS protein may serve for diagnostic purposes as biomarker [24]. Thus, these proteins can perfectly act as immunodiagnostic antigen [17] to detect $H$. contortus infection at early stage.

This study was designed to evaluate the diagnostic capacity of recombinant cold shock $H$. contortus protein (rHc-CS) and to detect specific antibodies during early and late $H$. contortus infections in goat using immunodiagnostic assays.

\section{Results \\ Purification, immunoblotting and early diagnostic potential}

The rHc-Cs was purified as Histidine-tagged fusion protein and resolved on 12\% SDS-PAGE which showed single band of about $38 \mathrm{kDa}$ (Fig. 1a). Immunoblotting results demonstrated that HcESPs could be recognized by anti- rHc-CS antibodies generated in Sprague Dawley (SD) rats. Furthermore, the native CS protein showed molecular mass of about $20 \mathrm{kDa}$ (Fig. 1b, Lane 1) and no antibody was detected with untreated rat sera (Fig. 1b, Lane 2). Moreover, immunoblotting results showed that initial antibodies were detected in sera of all artificially infected goats $(5 / 5)$ of group 1 collected at prepatent stage (14 D.P.I). The sera of all $H$. contortus-infected goats had detectable antigenicity at $21,35,49,63,85$, and 103 days of infection, no IgG antibody against rHcCS was detected in sera collected at day pre-infection and 7 D.P.I (Fig. 2). On the other hand, FECs showed that strongylid eggs were detected in all samples collected at 21 D.P.I (mean $\pm S E M=7.4 \pm 0.6), 28$ D.P.I (mean $\pm S E M=9.8 \pm 0.78$ ) and 35 D.P.I (mean $\pm S E M=$ $10.8 \pm 1.08)$ but the fecal samples collected at 0,7 and 14 D.P.I were negative for eggs. Furthermore, occurrence of $H$. contortus worms was confirmed at 30 D.P.I by necropsy in all tested goats. Moreover, the recombinant Hc-CS antigen did not show any cross-reactivity against goat sera infected with $T$. spiralis, $F$. hepatica and $T$. gondii (Fig. 3). These findings revealed that $\mathrm{rHc}-\mathrm{CS}$ protein had good immune-reactivity and antigenic characteristics which detected the specific antibody during early and late stages of $H$. contortus infection. Conversely, $H$. contortus infection was not detected by microscopic examination at early stage (7 and 14 D.P.I). 


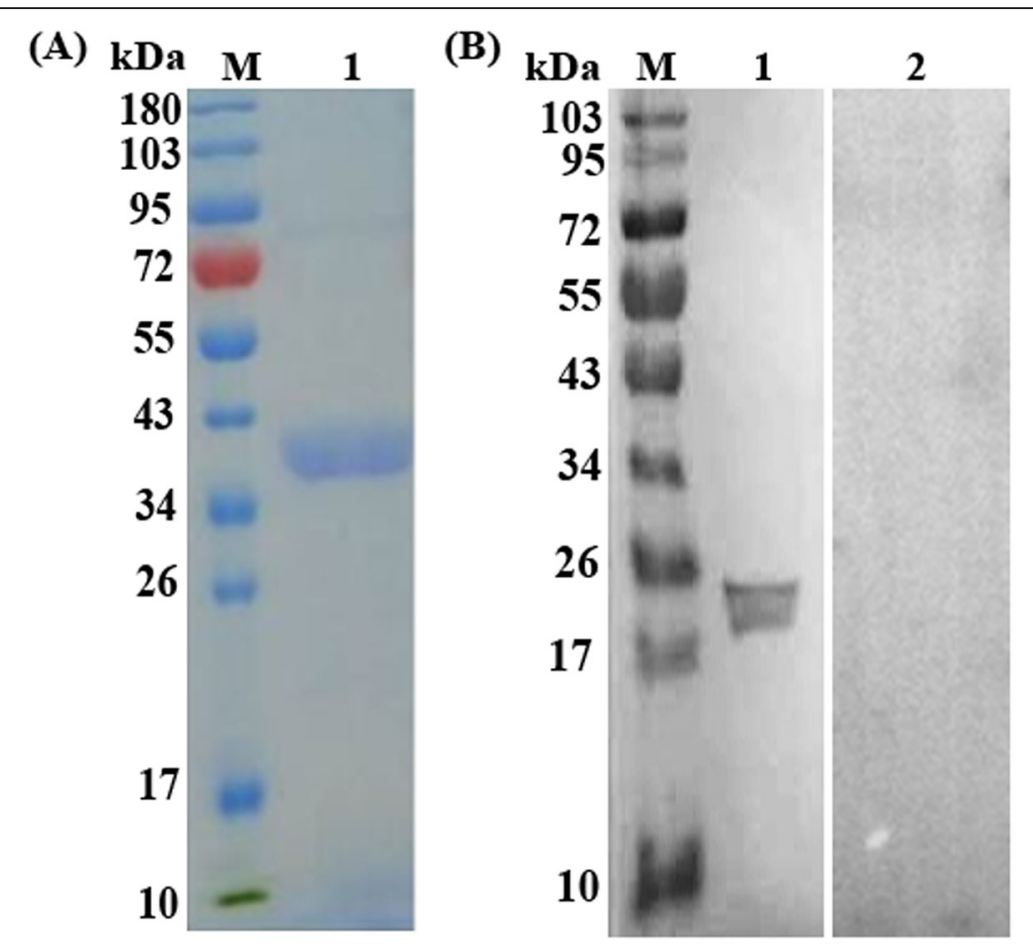

Fig. 1 Purification and immunoblotting of $\mathrm{rHc}-\mathrm{CS}$ protein. Lane M: standard protein pre stain molecular weight Marker. a Lane 1: Purified rHc-CS protein. $\mathbf{b}$ Lane 1: HcESPs were detected by rat anti-rHC-CS protein antibodies; Lane 2: membrane incubated with normal rat sera (as control)

\section{Optimization of rHc-CS indirect-ELISA}

Several format variables were evaluated to optimize the indirect-ELISA based on $\mathrm{rHc}-\mathrm{CS}$, utilizing a small set of four samples containing two known $H$. contortus positive and two known negative sera (group 3). The optimum antigen (rHc-CS) coating concentration and the optimum dilution ratio of serum were $0.28 \mu \mathrm{g} /$ well and 1:100 with the maximum $\mathrm{P} / \mathrm{N}\left(\mathrm{OD}_{450}=5.085\right)$ value, respectively (Table 1). Subsequently, the optimum incubating condition, blocking buffer, secondary antibody dilution, serum incubation time and blocking buffer incubation time were recorded as $37^{\circ} \mathrm{C} 1 \mathrm{~h}$ and overnight at $4{ }^{\circ} \mathrm{C}(\mathrm{P} / \mathrm{N}: 5.117$; Fig. 4a), 5\% Bovine Serum Albumin (BSA; $\mathrm{P} / N=5.222$; Fig. 4b), 1:4000 (P/N = 5.706: Fig. 4c), 60 min (Fig. 4d) and $120 \mathrm{~min}$ (Fig. 4e), respectively. Moreover, the best Tetramethylbenzidine (TMB) reactive time was recorded as 10 min (Fig. 4f). However, this optimized rHc-CS indirectELISA was performed to determine the checkerboard titration of all sera that were already categorized as either $H$. contortus positive or negative.

\section{Determination of the cut-off value}

To calculate the cut off value for negative sera in indirect ELISA, mean value $(0.244 \pm 0.004)$ was added into standard deviations (0.036) of OD values after multiplying by 3 as $3 \times 0.036$. OD values were obtained from 35 confirmed negative goat sera. The sera isolated from positive group showed significantly higher $\mathrm{OD}_{450}$ (>
$0.352)$ as compared to negative group (OD $<0.352)$. One way ANOVA was performed to observe significant differences $(P<0.05)$ in mean OD values among infected goats' sera by $H$. contortus $(0.645 \pm 0.012)$ and uninfected goats' sera $(0.244 \pm 0.004)$.

\section{Receiver operating characteristic (ROC)}

The influence of different cut-off values on the sensitivity and specificity of this antigen was investigated by ROC curve analysis. ROC analysis showed $100 \%$ sensitivity and specificity at cut-off value of 0.352 . Moreover, least difference was seemed in sensitivity $(96.8 \%)$ at cutoff value of 0.387 and specificity (96.8\%) at cut-off value of 0.316. The area under the ROC curve (AUC) was recorded as $1(P<0.001)$ with the cut-off value of 0.352 , which indicates high accuracy of the cut-off for classifying the serum samples into $H$. contortus positive or negative. Thus, the cut-off value for rHc-CS indirectELISA was selected as $\geq 0.352$ for further use to provide maximum sensitivity with very good specificity.

\section{Sero-diagnostic potential of rHc-CS indirect-ELISA}

The rHc-CS has definite diagnostic potential through the optimized indirect ELISA. The indirect-ELISA based on rHc-CS showed $100 \%$ sensitivity against $H$. contortus positive sera while $100 \%$ specificity against untreated goat sera. Furthermore, no false positive/negative results were observed. Total 33 known positive sera were analyzed 


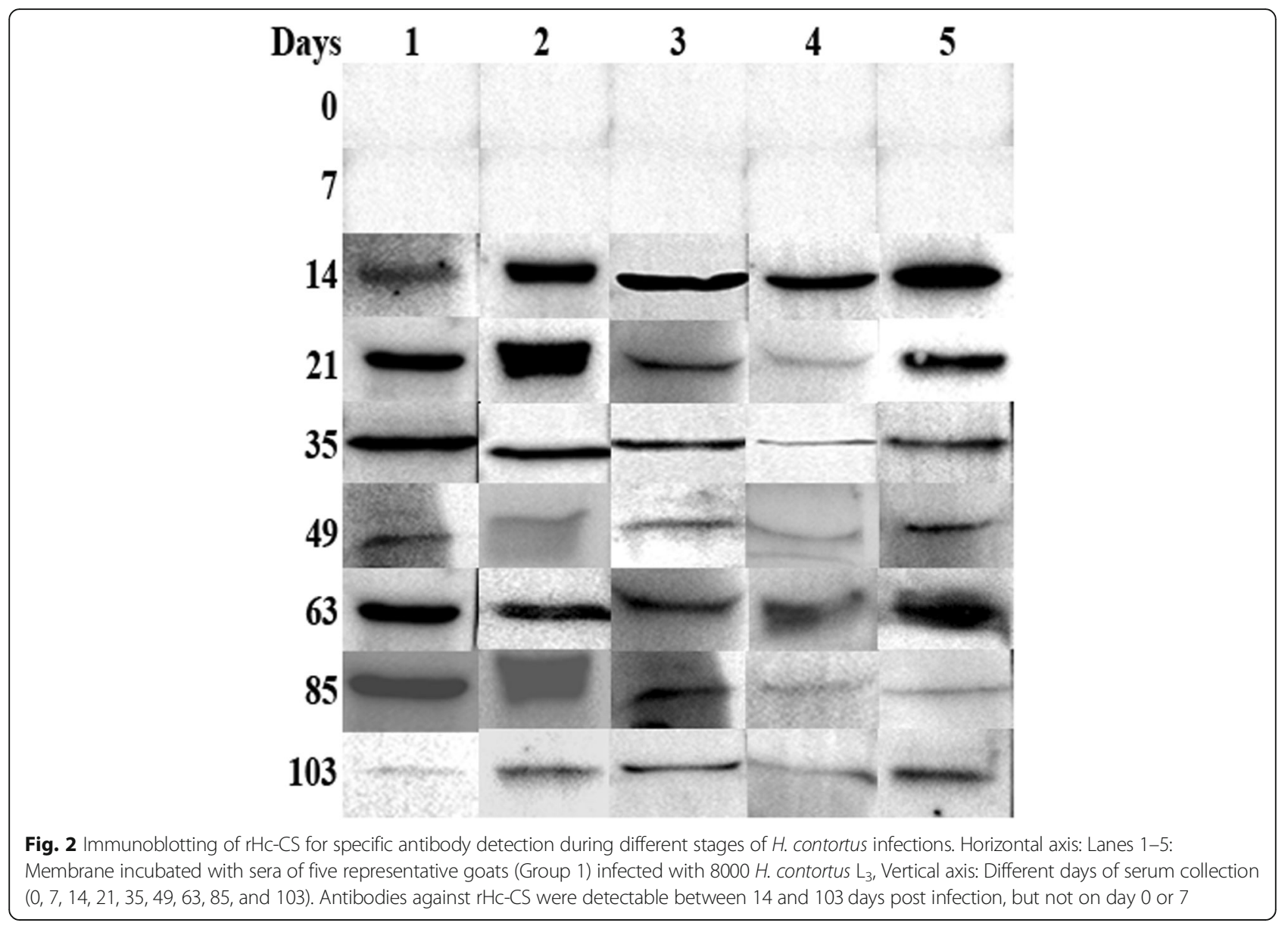

to evaluate the analytical sensitivity and $\mathrm{rHc}-\mathrm{CS}$ indirectELISA showed $100 \%$ sensitivity as all positive sera (33/33) were above the cut-off line (0.352). The analytical specificity was evaluated using sera positive for T. spiralis $(n=4), F$. hepatica $(n=4)$ and T. gondii $(n=4)$. Results of rHc-CS indirect-ELISA revealed that all sera positive for $T$. spiralis, F. hepatica, T. gondii and non-infected sera showed $\mathrm{OD}_{450}$ below the cut-off line. Scatter plot analysis showed significant differences $(P<0.001)$ between $H$. contortus infected sera and other parasites positive sera, while no significant difference $(P>0.05)$ was observed between helminths-free sera and other parasites' positive sera (Fig. 5). In order to diagnose haemonchosis in goats, $100 \%$ sensitivity of this antigen was measured and no negative control was higher than the cut-off point.

\section{Discussion}

$H$. contortus causes high mortality and morbidity rates in sheep and goats $[5,6]$. To control this infection efficiently, early diagnosis of the infection is inevitable as last two larval stages of this parasite feed on blood that loss up to one fifth of the total circulating erythrocyte volume from young animals and may cause fatal anemia [10]. Previous study reported the serological methods for diagnosis of Dicrocoelium dendriticium because these methods are more accurate in comparison to FEC test [25].

Recently, larval antigen, somatic antigens and crude antigen have been evaluated for the immunodiagnosis of $H$. contortus infection $[20,26,27]$. However, the specificity and sensitivity is compromised for these antigens. ESPs are released by parasites within the body of host that play vital role in pathogenesis and modulation of the immune response at the early stage of infection. It is further added that $H$. contortus interaction with host starts after the transition of $\mathrm{L}_{3}$ into $\mathrm{L}_{4}$ stage [28]. Furthermore, recombinant protein, having high immunoreactive concentration, may act as best immunodiagnostic antigen by achieving high sensitivity and specificity [29-36]. Recombinant HcCS is a major constituent of HcES proteins [23] which may have potential to diagnose $H$. contortus at early stage of infection. This study was designed to evaluate the specific antibodies detection during early and late infections of $H$. contortus using immunoblotting and indirect-ELISA based on rHc-CS in experimentally infected goat. In this study, rHc-CS was used as diagnostic antigen of $H$. contortus infection for detection of specific antibody during prepatent period and development of indirect ELISA as well. 


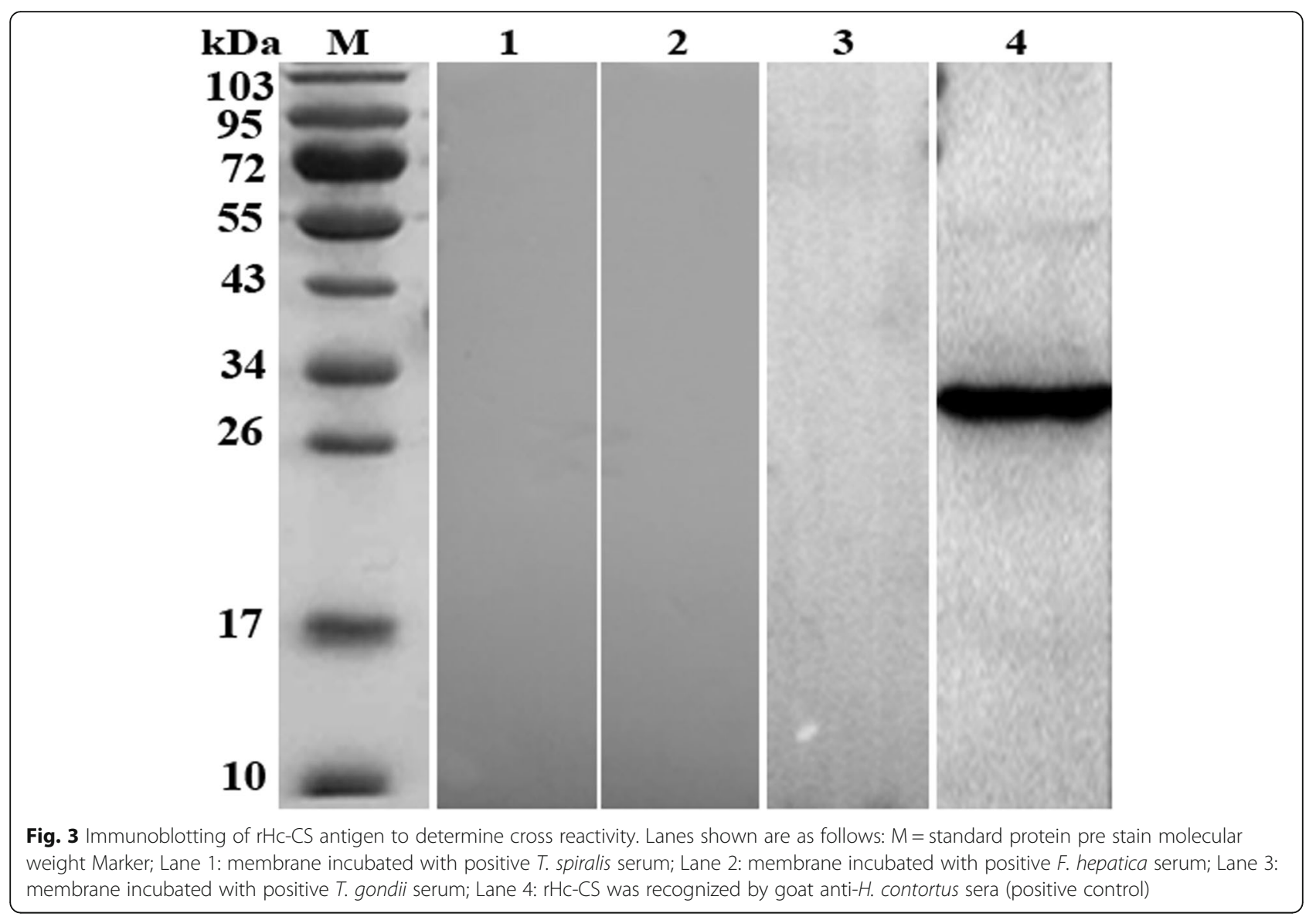

Table 1 Determination of the optimal rHc-CS coating concentration and serum dilution for indirect-ELISA

\begin{tabular}{|c|c|c|c|c|c|c|c|c|}
\hline \multirow{2}{*}{$\begin{array}{l}\text { Serum } \\
\text { Dilution }\end{array}$} & & \multicolumn{7}{|c|}{$\mathrm{OD}_{450}$ values of $\mathrm{rHc}-\mathrm{CS}$ at increasing coating concentrations } \\
\hline & & 0.07 & 0.14 & 0.28 & 0.56 & 1.12 & 2.25 & 4.5 \\
\hline \multirow[t]{3}{*}{$1: 25$} & (P) & 0.46 & 0.79 & 1.21 & 1.20 & 1.36 & 1.56 & 1.85 \\
\hline & $(\mathrm{N})$ & 0.19 & 0.30 & 0.35 & 0.53 & 0.64 & 0.94 & 1.61 \\
\hline & $\mathrm{P} / \mathrm{N}$ & 2.45 & 2.67 & 3.41 & 2.27 & 2.13 & 1.66 & 1.15 \\
\hline \multirow[t]{3}{*}{ 1:50 } & (P) & 0.40 & 0.68 & 1.19 & 1.18 & 1.21 & 1.49 & 1.63 \\
\hline & $(\mathrm{N})$ & 0.18 & 0.21 & 0.29 & 0.34 & 0.55 & 0.77 & 1.41 \\
\hline & $\mathrm{P} / \mathrm{N}$ & 2.20 & 3.21 & 4.05 & 3.45 & 2.21 & 1.94 & 1.15 \\
\hline \multirow[t]{3}{*}{ 1:100 } & (P) & 0.35 & 0.51 & 1.01 & 1.20 & 1.28 & 1.38 & 1.40 \\
\hline & (N) & 0.17 & 0.17 & 0.20 & 0.26 & 0.44 & 0.68 & 1.29 \\
\hline & $\mathrm{P} / \mathrm{N}$ & 2.07 & 2.88 & 5.08 & 4.57 & 2.91 & 2.01 & 1.08 \\
\hline \multirow[t]{3}{*}{ 1:200 } & $(\mathrm{P})$ & 0.23 & 0.41 & 0.78 & 0.86 & 0.92 & 0.97 & 1.22 \\
\hline & (N) & 0.17 & 0.13 & 0.16 & 0.22 & 0.30 & 0.50 & 1.00 \\
\hline & $\mathrm{P} / \mathrm{N}$ & 1.36 & 3.08 & 4.69 & 3.82 & 3.02 & 1.92 & 1.22 \\
\hline
\end{tabular}




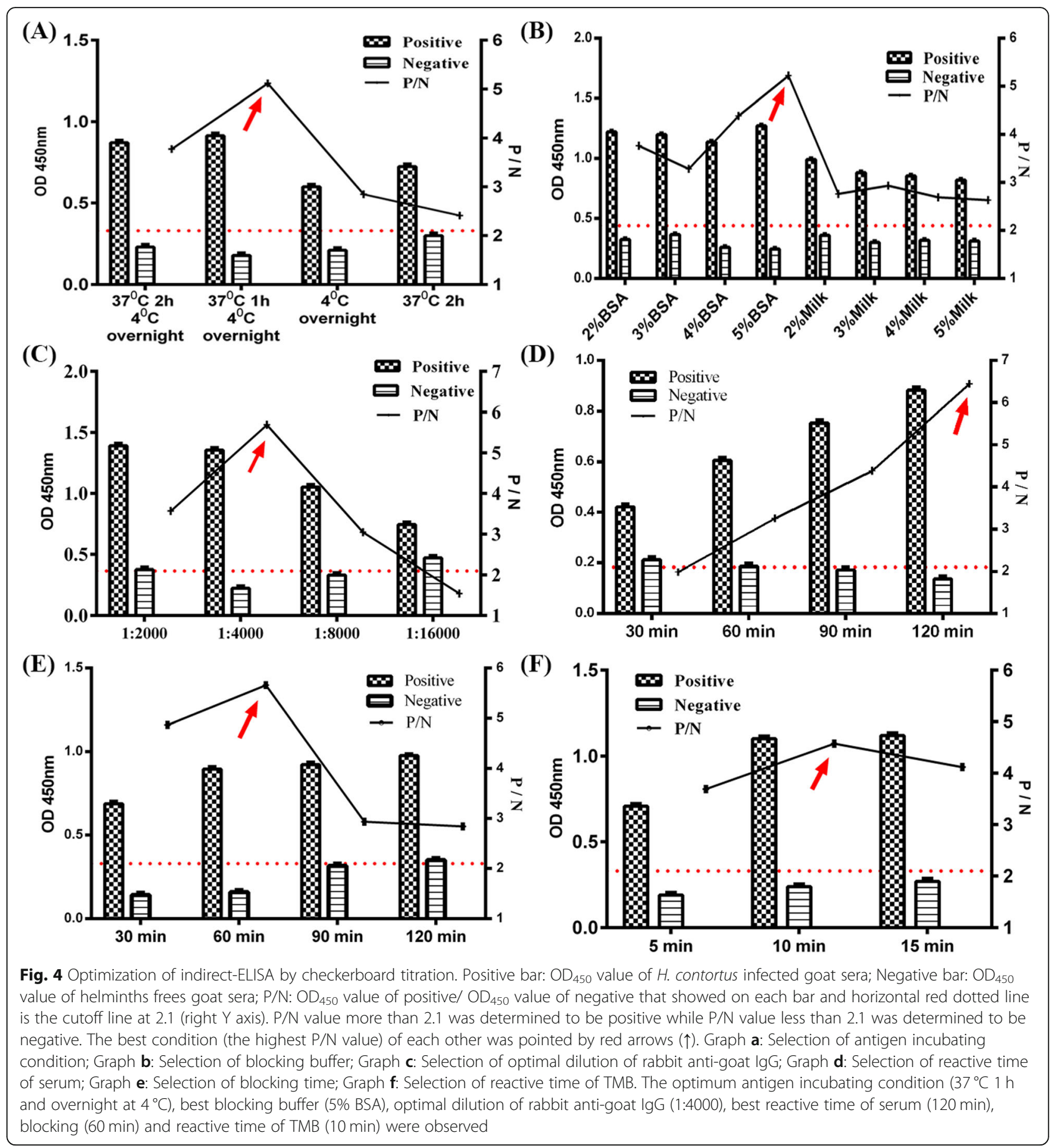

Immunoblotting is a useful technique to detect specific pathogen and antigen potency immunodiagnostic studies [37]. A confirmatory test for immunoblotting-positive sera was required to overcome the possible false positive results that may affect the certain diagnosis of infection [17]. In this study, immunoblotting results indicated that rHc-CS showed high immunoreactivity against polyclonal antibodies generated in sera of SD rats. The ELISA is a powerful tool as it provides a less time consuming, easy and safe way to perform serological detection. Therefore the association between ELISA and immunoblotting techniques can be seen as a potent way to increase sensitivity for immunodiagnosis purposes [38]. Previously, a study was conducted for prepatent detection of specific $H$. contortus antibody, early and late patency of $H$. contortus infections using ELISA based on Hc26 and immunoblotting in sheep [37]. Another study was performed using recombinant protein recIgE1-2 to detect $H$. contortus 


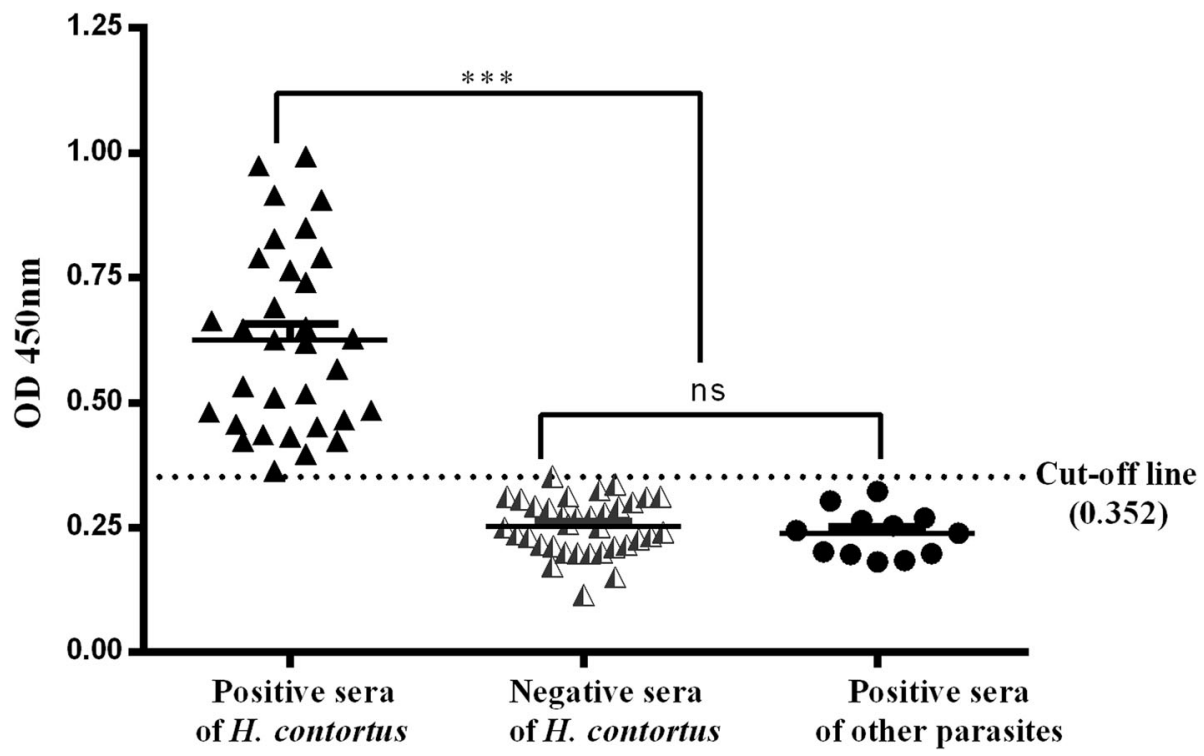

Fig. 5 Sensitivity, specificity and cross-reactivity of indirect-ELISA based on rHc-CS. The dotted horizontal line represents the cut-off value $\left(\mathrm{OD}_{450}=0.352\right)$ of indirect ELISA based on recombinant Hc-CS. Statistically significant differences $(P<0.001)$ were observed between $H$. contortuspositive sera and the other sera (T. spiralis, F. hepatica and T. gondii-positive and H. contortus-negative sera). No significant difference $(P>0.05)$ was noted between the $H$. contortus-negative and other parasites-positive serum samples

infection in sheep sera collected at 2 to 4 weeks post infection in which immunoblotting assay showed crossreactivity against sheep sera [39]. In contrast, the immunoblotting results of this study revealed that $\mathrm{rHc}-\mathrm{CS}$ antigen specifically reacted with antibodies present in all $H$. contortus positive goat sera at 14 D.P.I and persisted until 103 D.P.I. However, no anti-rHc-CS antibodies were detected in uninfected sera collected at day before infection. Previously, crude Excretory-Secretory (ES) protein of $H$. contortus-based Dot-ELISA was performed using sera collected at 1-3 weeks post infection sera but cross reactivity of this antigen with other nematode and trematode was not checked [15]. In contrast to this study, H. contortus antigen showed cross-reactivity against Fasciola hepatica and Moniezia expansa [40]. In previous study, somatic and adult larval antigen showed cross-reactivity with closely-related $H$. contortus species and possibly other genera [9]. In this study, rHc-CS did not show any cross-reactivity against coinfecting parasites (T. spiralis, $F$. hepatica and T. gondii). These results proved that specific-antibody detection has more specific and sensitive diagnostic potential as compared to conventional microscopic techniques. Thus, the evaluated $\mathrm{rHc}-\mathrm{CS}$ can be used as diagnostic antigen to detect specific antibodies effectively during prepatent period of $H$. contortus infection in goat. Antigenicity, sensitivity and specificity may be influenced by the secretion time of antigen. It has been reported earlier that rHc-CS releases at 14 and 60 D.P.I and antibodies against this molecule are produced [23]. Another possibe reason might be the type of antigen used in study.
Indirect ELISA based on rHc-CS was optimized and developed to complement the immunoblotting results. Previously, combined use of ELISA and immunoblotting has been reported as a powerful tool to immuno-diagnose different infections [38, 41]. In this study, rHc-CS indirectELISA showed higher diagnostic specificity and sensitivity as compared to somatic antigen-based indirect-ELISA format reported in previous study [20]. This contrast might be due to higher binding efficiency of rHc-CS antigen, as type of diagnostic antigens used in study may influence specificity, sensitivity and cross reactivity of assay [42]. In previous study, indirect ELISA using somatic and adult larval antigen results in relatively lower diagnostic specificity [9]. In present study, both sensitivity and specificity of the rHc-CS indirect-ELISA were measured as $100 \%$, that is similar to the findings of previous study in which His-ES24-based ELISA was performed to detect specific antibodies in $H$. contortus infected sera of sheep [17]. In contrast, 90\% [43] and $87 \%$ [41] diagnostic sensitivity of indirect ELISA was reported using different recombinant $H$. contortus tropomyosin antigens.

Various types of false negative and false positive reactions may influence the results, irrespective of antigens in indirect-ELISA [44]. To determine the background noise reactions, optimization of best antigen and antibodies dilution are crucial to increase the diagnostic potential of indirect ELISA. After standardization of $\mathrm{rHc}-\mathrm{CS}$ based indirect-ELISA, checkerboard titration results showed best performance with optimal coating concentration of antigen $(0.28 \mu \mathrm{g} /$ well), best serum dilution $(1: 100)$ and best 
working concentrations of secondary antibody (rabbit anti-goat IgG; 1:4000). Type of blocking buffers and their incubating time may also influence the performance of assay and may encourage false positive or false negative reactions [45]. In this study, to minimize this challenge of false reactions, different dilutions of different blocking buffers were optimized with different incubating times. Incubation time of buffer has significant effects on the performance of the assay. Therefore, the optimized results showed that the best blocking buffer was 5\% BSA which is same as the findings of previous study [46]. The optimum reactive time of blocking and TMB was recorded as 60 and $10 \mathrm{~min}$, respectively. Furthermore, the best $\mathrm{P} / \mathrm{N}$ value (6.44) was recorded when serum was incubated for 120 $\mathrm{min}$. To reduce time, serum can be incubated for $60 \mathrm{~min}$ because $\mathrm{P} / \mathrm{N}$ value was observed positive $(\mathrm{P} / N>2.1)$. This optimized rHc-CS based indirect-ELISA will be more authenticated tool for identifying goats in the prepatent stage of $H$. contortus infection than other antigen-based assays. The results of this study will facilitate the low cost early serological diagnosis of large number of animals and possibly vaccine development against the infection of $H$. contortus. This assay has high sensitivity and specificity for downstream application in field studies. However, further study is required to assess the cross-reactivity of rHc-CS against other nematodes belonging to Trichostrongylidae family. Additionally, to fine tune this assay epidemiological surveillance studies with huge number of samples are required.

The rHc-CS antigen based indirect-ELISA will be pivotal tool for farmers to identify $H$. contortus infection even at early stage in goat. For this purpose, veterinarian will be called at farms to collect blood for further analyses. After 10 days, blood samples will be collected again from the goats with negative results for confirmation.

\section{Conclusions}

Combine use of immunoblotting and indirect ELISA showed that rHc-CS is a specific, sensitive, and potential immunodiagnostic antigen which may consistently detect $H$. contortus antibodies in goats during early as well as late infection of $H$. contortus.

\section{Methods}

\section{Ethical statement}

All experimental protocols were approved by the Science and Technology Agency of Jiangsu Province (Approval ID: SYXK (SU) 2010-0005).

\section{Study population}

Local crossbred goats ( $n=35$, age $\simeq 6$ months) were bought from a farm in Xuyu city, Jiangsu, China and kept under controlled conditions in animal house of Nanjing Agricultural University (NAU). All goats were orally dewormed twice with Levamisole $(8 \mathrm{mg} / \mathrm{kg})$ at two weeks interval to remove natural parasitic infections. Fecal samples were collected from all goats twice a week and analyzed microscopically for helminth eggs. Helminthes free goats were utilized for further experiment, After 25 days of first deworming, goats were divided randomly into group 1 $(n=5)$, group $2(n=28)$ and group $3(n=2)$.

Female SD rats of $150 \mathrm{~g}$ body weight $(n=6)$ were obtained from the Experimental Animal Center of Jiangsu, PR China (Certified: SCXK 2008-0004). Rats were separated randomly into two groups, group $1(n=3)$ and group $2(n=3)$ to collect polyclonal antibodies. Rats were kept in sterilized room with free access of food and water. Anti-rHc-CS polyclonal antibodies were collected following the methods as described early [47]. Briefly, complete Freund's adjuvant was equally mixed with rHc-CS protein $(300 \mu \mathrm{g})$ and injected subcutaneously in SD rats of group 1. After 14 days, incomplete Freund's adjuvant mixed equally with $\mathrm{rHc}-\mathrm{CS}$ protein was injected twice with 1 week interval. Finally, SD rats were anesthetized with 25\% isoflurane (inhaling anesthesia) after 1 week of the last dose by open drop method [48]. Group 2 was kept untreated as control. Rats were euthanized by head dislocation after collecting blood from eye to prepare sera.

\section{H. contortus infective larva $\left(\mathrm{L}_{3}\right)$}

The $H$. contortus strain was maintained by serial passages in helminth-free goats, at MOE laboratory NAU. H. contortus $\left(\mathrm{L}_{3}\right)$ used in these experiments were obtained from feces of infected goats using conventional method [49]. Briefly, feces from $H$. contortus infected-goat were collected, crushed, mixed with water and combined with vermiculite to keep mixture moist at room temperature. The pan was covered with aluminum foil having several holes to allow air flow. After 10 days, mixture was filtered through cheesecloth to collect larvae to examine microscopically that were preserved at $4{ }^{\circ} \mathrm{C}$ in penicillin $\mathrm{G}$ mixed with water until use.

\section{Experiment 1}

First experiment was performed to assess early diagnostic potential of rHc-CS protein during different stages of $H$. contortus infection using immunoblotting assay. For this purpose, group $1(n=5)$ was artificially infected with 8000 infected larva of $H$. contortus $\left(\mathrm{L}_{3}\right)$ orally and group $3(n=2)$ was kept uninfected as control. Serum samples were collected from group 1 (infected) and group 3 (uninfected) at specified days; one day prior challenging infection and 7 to 103 days post challenging infection with week interval for antibody detection.

Moreover, McMaster egg count technique was performed to examine the fecal samples collected at 7, 14, 21, 28 and 35 D.P.I as described previously [50]. 


\section{Experiment 2}

Experiment 2 was performed to develop and optimize indirect ELISA based on rHc-CS. In this regard, Group 2 $(n=28)$ was orally infected with $8000 \mathrm{H}$. contortus $\mathrm{L}_{3}$ and fecal examination was performed to confirm $H$. contortus infection before collecting the sera. Moreover, at 30 D.P.I the goats of group 2 were euthanized by injecting sodium pentobarbital (> $150 \mathrm{mg} / \mathrm{kg}$ ) intravenously [48]. Furthermore, necropsy was performed to confirm the presence of adult worms embedded in the abomasum.

\section{Purification of recombinant Hc-CS}

Recombinant plasmid (pET-32a + rHc-CS, GenBank: CDJ84294.1) was provided by MOE joint international Research Laboratory, Preventive Veterinary Medicine, NAU and protein was purified by following standard protocol [47]. After transformation of recombinant plasmid into E.coli BL21 (DE3), product was cultured in ampicillin containing LB (Luria Bertani) medium. Ni2+ nitrilotriacetic acid column (GE Healthcare, USA) was used to purify recombinant protein following the manufacturer's instructions. The quantification of rHc-CS protein was analyzed by Bradford method [51] and protein was detoxified using Toxin Eraser $^{\mathrm{Tm}}$ Endotoxin Removal kit (GeneScript, USA).

\section{Immunoblotting assay}

Immunoblotting is a method of choice for $H$. contortus infection. It helps to select target protein for the diagnostic purposes as well as for the antigenic immunogenicity and immunoreactivity evaluation [52]. Immunoblotting was performed to evaluate the immunoreactivity of $\mathrm{rHc}-\mathrm{CS}$ using rat sera following the methods as described in our previous study [41]. Moreover, immunoblotting analysis was also performed to evaluate the antigenic characteristics of rHc-CS at early stage as well as late stage of $H$. contortus infection in sera of experimentally infected goats (Group 1). Upon each blood sampling day, the whole immunoblotting practice was repeated whereas the primary antibody differed. All anti $H$. contortus sera taken from infected (Group 1) and uninfected goats (Group 3), on those sampling days were taken as the primary antibody. Moreover, the specificity of rHc-CS against infected sera of Fasciola hepatica (F. hepatica), Trichinella spiralis (T. spiralis) and Toxoplasma gondii (T. gondii) was also checked by immunoblotting.

\section{Optimization and development of indirect-ELISA}

Indirect-ELISA was performed to assess the immunodiagnostic potential of $\mathrm{rHc}-\mathrm{CS}$ [53]. Indirect-ELISA based on rHc-CS was optimized by evaluation of numerous format variables including working dilution of $\mathrm{rHc}-\mathrm{CS}$ antigen (4.5 to $0.07 \mu \mathrm{g} /$ well), serum samples (1:25 to 1 : 200 dilutions), secondary antibody HRP conjugated rabbit anti-goat IgG (1:2000 to $1: 16000)$ and working time of antigen coating $\left(37^{\circ} \mathrm{C} 2 \mathrm{~h}-4{ }^{\circ} \mathrm{C}\right.$ overnight, $37^{\circ} \mathrm{C} 1 \mathrm{~h}-4{ }^{\circ} \mathrm{C}$ overnight), blocking (30-120 min), serum incubation (30$120 \mathrm{~min})$ and $\mathrm{TMB}$ reaction $(5-15 \mathrm{~min})$. Furthermore, best blocking buffer (2, 3, 4, 5\% BSA and 2, 3, 4, 5\% Milk) was also determined using same method. Hence, checkerboard titration was used to derive OD values and calculated as the positive to negative $(\mathrm{P} / \mathrm{N})$ ratio [54].

In order to evaluate the diagnostic potential and to complement the immunoblotting results, standardized indirect-ELISA was developed as described previously $[41,55]$. Moreover, serum samples $(n=33)$ from infected goats were used to calculate diagnostic sensitivity and serum samples of all goats before artificial infection $(n=$ 35 ) were used to calculate diagnostic specificity of rHcCS based indirect ELISA using following formula [56].

$$
\begin{aligned}
& \text { Sensitivity }=\frac{\text { True Positive }}{\text { True Positive }+ \text { False Negative }} \\
& \text { Specificity }=\frac{\text { True Negative }}{\text { True Negative }+ \text { False Positive }}
\end{aligned}
$$

Additionally, to confirm the cross-reactivity against commonly found parasites, a total of 12 goat serum samples (4 samples for each parasite, Kept in the MOE laboratory of NAU) against F. hepatica, T. spiralis and $T$. gondii were used. All the experiments were performed in duplicate.

\section{Determination of cut-off value}

Sera $(n=35)$ collected from all goats before $H$. contortus infection were used to determine cut-off value. The cutoff value was calculated by taking "mean absorbance values of known negative sera + (3× standard deviation)" [57]. $\mathrm{OD}_{450}$ value of sera greater than cut-off value was considered as sero-positive and $\mathrm{OD}_{450}$ value below cutoff value was considered as sero-negative [34].

\section{Statistical analysis}

ROC analysis was used to simulate the influence of different cut-off values on sensitivity and specificity of the test [38]. ROC curves were obtained using statistical software MedCalc (version 15; http://www.medcalc.be). Statistical analysis of data was assessed by using software (Graph Pad Prism ${ }^{\mathrm{TM}}$ v6 07).

\footnotetext{
Abbreviations

AEC: Animal Ethics Committee; BSA: Bovine serum albumin; D.P.I: Days post infection; HcESPS: Excretory and secretory products of Haemonchus contortus; HRP: Horseradish peroxidase; IPTG: Isopropyl-b-D-thiogalactopyranoside; kDa: Kilodalton; MW: Molecular weight; NAU: Nanjing Agricultural University; OD: Optical density; P/N: Positive to negative; PBMCs: Peripheral blood mononuclear cells; PVDF: Polyvinyl difluoride membrane; $r \mathrm{HC}^{-}$ CS: Recombinant Cold Shock domain containing protein; ROC: Receiveroperating characteristics; SD: Sprague Dawley; SDS-PAGE: Sodium dodecyl sulfate-polyacrylamide gel electrophoresis; TBS-T: Tris-Buffered Saline containing 0.05\% Tween 20; TMB: Tetramethylbenzidine
} 


\section{Acknowledgements}

We gratefully thank to Syed Sajjad Hussain Naqvi, Syeda Sughra Khatoon Naqvi and Muhammad Abbas, Layyah, Punjab, Pakistan.

\section{Authors' contributions}

YRF directed the project and participated in the coordination and management of the study. MAHN performed the laboratory tests and the data analysis and wrote the manuscript. KA and SZN provided some ideas for the experimental design. MAM and CW helped in blood sampling. MWH and SAL helped in parasite collection. LXR, SXK and XLX provided new analytical reagents and tools. All authors read and approved the final manuscript.

\section{Authors' information}

Not applicable.

\section{Funding}

This work was supported by the National Key Research and Development Program of China (2017YFD0501200), the National Natural Science Foundation of China (31872464), the National Key Basic Research Program (973 Program) of China (2015CB150300) and Priority Academic Program Development of Jiangsu Higher Education Institutions (PAPD). The funding bodies played no role in the design of the study, the collection, analysis, and interpretation of data and in writing the manuscript.

\section{Availability of data and materials}

All data generated or analyzed during this study are included in this published article.

\section{Ethics approval and consent to participate}

The study was conducted following the guidelines of the Animal Ethics Committee, Nanjing Agricultural University (NAU), China. All experimental protocols were approved by the Science and Technology Agency of Jiangsu Province (Approval ID: SYXK (SU) 2010-0005).

\section{Consent for publication}

Not applicable.

\section{Competing interests}

The authors declare that they have no competing interests.

Received: 23 June 2019 Accepted: 24 January 2020

Published online: 03 February 2020

\section{References}

1. Roberts L, Janovy JJ. Phylum Nematoda: form, fuction and classification. In: Foundations of Parasitology. Dubuque USA Wm C Brown Publ; 1996. p. 355-84.

2. Urquhart GM, Armour J, Dunca JL, Dunn A, Jennings FW. Veterinary parasitology, 2nd Edition. Blackwell Sci Ltd London. 2000.

3. Soulsby EJL. Helminthes, arthropods and Protozoa of domesticated animals. Bailliere Tindall, London. 1982;7:74-85.

4. Schallig HDFH, Hornok S, Cornelissen JBWJ. Comparison of two enzyme immunoassays for the detection of Haemonchus contortus infections in sheep. Vet Parasitol. 1995;57:329-38.

5. Tariq KA, Chishti MZ, Ahmad F, Shawl AS. Epidemiology of gastrointestinal nematodes of sheep managed under traditional husbandry system in Kashmir valley. Vet Parasitol. 2008:158:138-43.

6. Nwosu CO, Madu PP, Richards WS. Prevalence and seasonal changes in the population of gastrointestinal nematodes of small ruminants in the semiarid zone of North-Eastern Nigeria. Vet Parasitol. 2007;144:118-24.

7. Aziz MA. Present status of the world goat populations and their productivity. Lohmann Inf. 2010;45:42-52.

8. Wang C, Li F, Zhang Z, Yang X, Ahmad AA, Li X, et al. Recent research progress in China on Haemonchus contortus. Front Microbiol. 2017:8:1-11.

9. Lone BA, Chishti MZ, Ahmad F, Tak H, Hassan J. Immunodiagnosis of Haemonchus contortus infection in sheep by indirect enzyme linked immunosorbent assay (ELISA). Iran J Vet Res Shiraz Univ. 2012;13:49-53.

10. Clark CH, Kiesel GK, Goby CH. Measurement of blood loss caused by Haemonchus contortus. Am J Vet Med. 1962;23:977-80.
11. Colditz IG, Le Jambre LF. Development of a faecal occult blood test to determine the severity of Haemonchus contortus infections in sheep. Vet Parasitol. 2008;153:93-9.

12. Kandil OM, Hendawy SHM, El AH, Cross ÁEÁ. Evaluation of different Haemonchus contortus antigens for diagnosis of sheep haemonchosis by ELISA and their cross reactivity with other helminthes. J Parasit Dis. 2017:41:678-83.

13. Kaplan RM, Burke JM, Terrill TH, Miller JE, Getz WR, Mobini S, et al. Validation of the FAMACHAC eye color chart for detecting clinical anemia in sheep and goats on farms in the southern United States. Vet Parasitol. 2004:123:105-20.

14. Gasser RB, Bott NJ, Chilton NB, Hunt P, Beveridge I. Toward practical, DNAbased diagnostic methods for parasitic nematodes of livestock - bionomic and biotechnological implications. Biotechnol Adv. 2008;26:325-34.

15. Prasad A, Nasir A, Singh N. Detection of anti-Haemonchus contortus antibodies in sheep by dot- ELISA with immunoaffinity purified fraction of ES antigen during prepatency. Indian J Exp Biol. 2008;46:94-9.

16. Yatsuda AP, Krijgsveld J, Cornelissen AWCA, Heck AJR, De Vries E. Comprehensive analysis of the secreted proteins of the parasite Haemonchus contortus reveals extensive sequence variation and differential immune recognition. J Biol Chem. 2003;278:16941-51.

17. Li X, Du A, Cai W, Hou Y, Pang L, Gao X. Evaluation of a recombinant excretory secretory Haemonchus contortus protein for use in a diagnostic enzyme-linked immunosorbent assay. Exp Parasitol. 2007;115:242-6.

18. Cox GN, Pratt D, Hageman R, Boisvenue RJ. Molecular cloning and primary sequence of a cysteine protease expressed by Haemonchus contortus adult worms. Mol Biochem Parasitol. 1990;41:25-34.

19. Gomez-Muñoz MT, Cuquerella M, Alunda JM. Identification and partial purification of a 26 kilodalton antigen of adult Haemonchus contortus. Int J Parasitol. 1996:26:311-8

20. Gowda AKJ. Sero-prevalence of Haemonchus contortus infection in sheep by indirect-ELISA using somatic antigen. J Parasit Dis. 2016:40:464-8.

21. Roeber F, Jex AR, Gasser RB. Advances in the diagnosis of key gastrointestinal nematode infections of livestock, with an emphasis on small ruminants. Biotechnol Adv. 2013;31:1135-52.

22. Lindquist JA, Mertens PR. Cold shock proteins: from cellular mechanisms to pathophysiology and disease. Cell Commun Signal. 2018;16:1-14.

23. Gadahi JA, Wang S, Bo G, Ehsan M, Yan R, Song X. Proteomic Analysis of the Excretory and Secretory Proteins of Haemonchus contortus ( HcESP) Binding to Goat PBMCs In Vivo Revealed Stage-Specific Binding Profiles. PLoS One. 2016:1-26. https://doi.org/10.1371/journal.pone.0159796.

24. Rohr I, Braicu El, En-Nia A, Heinrich M, Richter R, Chekerov R, et al. Y-box protein-1/p18 as novel serum marker for ovarian cancer diagnosis: a study by the tumor Bank ovarian Cancer (TOC). Cytokine. 2016:85:157-64.

25. Naeemipour M, Hashemitabar GR, Dastjerdi K, Mojaver MJ, Mohammadi HR. Comparison of fecal egg counts and ELISA for the diagnosis of Dicrocoelium Dendriticum infection. Pol J Vet Sci. 2016;19:573-80.

26. Kandil OM, Eid A, Elakabawy LM, HMA AKA. Immunodiagnostic Potency of Different Haemonchus contortus Antigens for Diagnosis of Experimentally and Naturally Haemonchosis in Egyptian Sheep. Acta Parasitol Glob. 2015:6:238-47.

27. Mohmad A, Chandra D, Saravanan BC, HVM, Vinodh VK, Fular A, et al. Development of a recombinant TaSP-based Dot-ELISA for detection of Theileria annulata infection in cattle. Ticks Tick Borne Dis. 2018;9:1416-20.

28. Laing R, Kikuchi T, Martinelli A, Tsai IJ, Beech RN, Redman E, et al. The genome and transcriptome of Haemonchus contortus, a key model parasite for drug and vaccine discovery. Genome Biol. 2013;14:R88. https://doi.org/ 10.1186/gb-2013-14-8-r88.

29. He H, Li Y, Liu J, Liu Z, Yang J, Liu A, et al. An indirect ELISA for detection of Theileria spp. antibodies using a recombinant protein ( $r$ TISP) from Theileria luwenshuni. Exp Parasitol. 2016;166:89-93.

30. Faramarzi T, Mobasheri M, Yoosefy A, Valadkhani Z. Expression and purification of truncated recombinant B8/1 protein of Echinococcus granulosus for diagnosis of hydatid infection in human. Acta Trop. 2019;191:139-45.

31. Shrestha A, Freudenschuss B, Schwarz L, Joachim A. Development and application of a recombinant protein-based indirect ELISA for the detection of serum antibodies against Cystoisospora suis in swine. Vet Parasitol. 2018:258:57-63.

32. Wang Y, Nie H, Gu X, Wang T, Huang X, Chen L, et al. An ELISA using recombinant TmHSP70 for the diagnosis of Taenia multiceps infections in goats. Vet Parasitol. 2015;212:469-72. 
33. Zhuo X, Yu Y, Chen X, Zhang Z, Yang Y, Du A. Development of a colloidal gold immunochromatographic strip based on HSP70 for the rapid detection of Echinococcus granulosus in sheep. Vet Parasitol. 2017;240:34-8.

34. Mandal M, Banerjee PS, Kumar S, Garg R, Ram H, Kundu K, et al. Development and evaluation of serodiagnostic assays with recombinant BgSA1 of Babesia gibsoni. Vet Parasitol. 2014;205:483-9.

35. Feng J, Xu R, Zhang X, Han Y, He C, Lu C, et al. A candidate recombinant antigen for diagnosis of Schistosomiasis japonica in domestic animals. Vet Parasitol. 2017;243:242-7.

36. Beck ST, Leite OM, Arruda RS, Ferreira AW. Combined use of Western blot/ ELISA to improve the serological diagnosis of human tuberculosis. Braz J Infect Dis. 2005;9:35-43.

37. Gómez-Muñoz MT, Domínguez IA, Gómez-Iglesias LA, Fernández-Pérez FJ, Méndez S, de la Fuente C, et al. Serodiagnosis of haemonchosis with a somatic antigen (Hc26) in several breeds of sheep. J Vet Diagn Investig. 2000;12:354-60.

38. Finger PF, Pepe MS, Dummer LA, Magalhães CG, De CCC, SDO H, et al. Combined use of ELISA and Western blot with recombinant $N$ protein is a powerful tool for the immunodiagnosis of avian infectious bronchitis. Viro J. 2018;15:189

39. Kooyman FNJ, Van Kooten PJS, Huntley JF, MacKellar A, Cornelissen AWCA Schallig HDFH. Production of a monoclonal antibody specific for ovine immunoglobulin $\mathrm{E}$ and its application to monitor serum lgE responses to Haemonchus contortus infection. Parasitology. 1997;114:395-406.

40. Kandil OM, Hendawy SHM, El Namaky AH, Gabrashanska MP, Nanev VN Evaluation of different Haemonchus contortus antigens for diagnosis of sheep haemonchosis by ELISA and their cross reactivity with other helminthes. J Parasit Dis. 2017;41:678-83.

41. Naqvi MA, Naqvi SZ, Memon MA, Aimulajiang K, Haseeb M, Xu L, et al. Combined Use of Indirect ELISA and Western Blotting with Recombinant Hepatocellular Carcinoma-Associated Antigen 59 Is a Potential Immunodiagnostic Tool for the Detection of Prepatent Haemonchus contortus Infection in Goat. Animals (Basel). 2019;9:548.

42. Waritani T, Chang J, McKinney B, Terato K. An ELISA protocol to improve the accuracy and reliability of serological antibody assays. MethodsX. 2017:4:153-65.

43. Naqvi MA. UI H, Jamil T, Naqvi SZ, Memon MA, Aimulajiang K, Aleem MT, et al. Immunodiagnostic potential of recombinant tropomyosin during prepatent Haemonchus contortus infection in goat. Res Vet Sci. 2020;128: 197-204.

44. Zhang W. Cheng B bei, Chen S, Wang M shu, Jia R yong, Zhu D Kang, et al. Development and optimization of a double antibody sandwich ELISA for the detection of goose T cell surface CD8a molecule. J Integr Agric. 2016; 15:2363-8.

45. Ammida NHS, Micheli L, Palleschi G. Electrochemical immunosensor for determination of aflatoxin B 1 in barley. Anal Chim Acta. 2004;520:159-64.

46. Xiao Y, Isaacs SN. Enzyme-linked Immunosorbent assay (ELISA) and blocking with bovine serum albumin (BSA) - not all BSAs are alike. J Immunol Methods. 2013;384:148-51.

47. Naqvi MA, Memon MA, Jamil T, Naqvi SZ, Aimulajiang K, Gadahi JA, et al. Galectin Domain Containing Protein from Haemonchus contortus modulates the immmune functions of goat PBMCs and regulates CD4 + T- Helper cells in vitro. Biomolecules. 2020;10:116.

48. Flecknell P, Lofgren JLS, Dyson MC, Marini RR, Michael Swindle M, Wilson RP. Preanesthesia. Analgesia, and Euthanasia. Laboratory Animal Medicine: Anesthesia; 2015.

49. Gadahi JA, Yongqian B, Ehsan M, Zhang ZC, Wang S, Yan RF, et al. Haemonchus contortus excretory and secretory proteins (HcESPs) suppress functions of goat PBMCs in vitro. Oncotarget. 2016;7:35670-9.

50. Ljungström S, Melville L, Skuce PJ, Höglund J. Comparison of Four Diagnostic Methods for Detection and Relative Quantification of Haemonchus contortus Eggs in Feces Samples. Front Vet Sci. 2018;4:239.

51. Han K, Xu L, Yan R, Song X, Li X. Cloning, expression and characterization of $\mathrm{NAD}+$-dependent glyceraldehyde-3-phosphate dehydrogenase of adult Haemonchus contortus. J Helminthol. 2011;85:421-9.

52. El-Hassan EM, El-Bahr SM. Antigenic and immunogenic components of Haemonchus longistipes identified by western Immunobloting. Am J Biochem Biotechnol. 2013:8:164-70

53. Crowther JR. Systems in ELISA. Methods Mol Biol. 2000;149:9-44.

54. Tankaew $P$, Singh-La T, Titaram C, Punyapornwittaya V, Vongchan P, Sawada $T$, et al. Evaluation of an in-house indirect ELISA for detection of antibody against Haemorrhagic septicemia in Asian elephants. J Microbiol Methods. 2017;134:30-4.

55. Tankaew P, Srisawat W, Singhla T, Tragoolpua K, Kataoka Y, Sawada T, et al. Comparison of two indirect ELISA coating antigens for the detection of dairy cow antibodies against Pasteurella multocida. J Microbiol Methods. 2018;145:20-7.

56. Deo VK, Inagaki Y, Murhandarwati EH, Asmara W, Miyazaki T, Kato T, et al. Sero-diagnostic potential of Plasmodium falciparum recombinant merozoite surface protein (MSP)-3 expressed in silkworm. Parasitol Int. 2019;72:101938.

57. Anuracpreeda P, Chawengkirtikul R, Tinikul Y, Poljaroen J, Chotwiwatthanakun C, Sobhon P. Diagnosis of Fasciola gigantica infection using a monoclonal antibody-based sandwich ELISA for detection of circulating cathepsin B3 protease. Acta Trop. 2013;127:38-45.

\section{Publisher's Note}

Springer Nature remains neutral with regard to jurisdictional claims in published maps and institutional affiliations.
Ready to submit your research? Choose BMC and benefit from:

- fast, convenient online submission

- thorough peer review by experienced researchers in your field

- rapid publication on acceptance

- support for research data, including large and complex data types

- gold Open Access which fosters wider collaboration and increased citations

- maximum visibility for your research: over $100 \mathrm{M}$ website views per year

At BMC, research is always in progress.

Learn more biomedcentral.com/submissions 